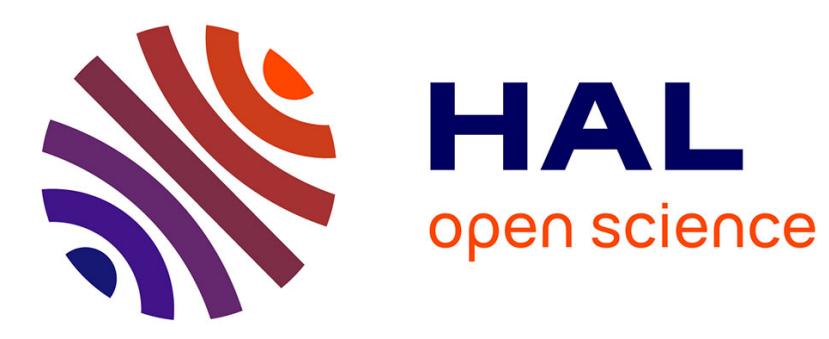

\title{
Recovery of Cosparse Signals with Greedy Analysis Pursuit in the Presence of Noise
}

Sangnam Nam, Mike Davies, Michael Elad, Rémi Gribonval

\section{To cite this version:}

Sangnam Nam, Mike Davies, Michael Elad, Rémi Gribonval. Recovery of Cosparse Signals with Greedy Analysis Pursuit in the Presence of Noise. CAMSAP - 4th International Workshop on Computational Advances in Multi-Sensor Adaptive Processing - 2011, Dec 2011, San Juan, Puerto Rico. hal-00691162

\section{HAL Id: hal-00691162 \\ https://hal.inria.fr/hal-00691162}

Submitted on 25 Apr 2012

HAL is a multi-disciplinary open access archive for the deposit and dissemination of scientific research documents, whether they are published or not. The documents may come from teaching and research institutions in France or abroad, or from public or private research centers.
L'archive ouverte pluridisciplinaire HAL, est destinée au dépôt et à la diffusion de documents scientifiques de niveau recherche, publiés ou non, émanant des établissements d'enseignement et de recherche français ou étrangers, des laboratoires publics ou privés. 


\title{
Recovery of Cosparse Signals with Greedy Analysis Pursuit in the Presence of Noise
}

\author{
(Invited Paper) \\ Sangnam Nam*, Michael E. Davies ${ }^{\dagger}$, Michael Elad ${ }^{\ddagger}$ and Rémi Gribonval* \\ ${ }^{*}$ Centre de Recherche INRIA Rennes - Bretagne Atlantique, 35042 Rennes, France \\ ${ }^{\dagger}$ School of Engineering and Electronics, The University of Edinburgh, Edinburgh, EH9 3JL, UK \\ $\ddagger$ Department of Computer Science, The Technion, Haifa 32000, Israel
}

\begin{abstract}
The sparse synthesis signal model has enjoyed much success and popularity in the recent decade. Much progress ranging from clear theoretical foundations to appealing applications has been made in this field. Alongside the synthesis approach, an analysis counterpart has been used over the years. Despite the similarity, markedly different nature of the two approaches has been observed. In a recent work, the analysis model was formally formulated and the nature of the model was discussed extensively. Furthermore, a new greedy algorithm (GAP) for recovering the signals satisfying the model was proposed and its effectiveness was demonstrated.

While the understanding of the analysis model and the new algorithm has been broadened, the stability and the robustness against noise of the model and the algorithm have been mostly left out. In this work, we adapt and propose a new GAP algorithm in order to deal with the presence of noise. Empirical evidence for the algorithm is also provided.
\end{abstract}

\section{INTRODUCTION}

In many tasks in image and signal processing, only limited-sometimes highly insufficient—data for the signals of interest are available. Even when sufficient amount of observations is available, the data can frequently be corrupted by noise. Adding on top of these the case where the data are both incomplete and corrupted by noise, one would be tempted to simply abandon any hope of performing reasonable work on those signals.

Fortunately, the signals that we are interested in are not just 'ordinary random signals.' They exhibit highly regular behavior among them. When we mathematically model these properties shared by the signals of interest, we arrive at sometimes spectacular results even for the problems with initial bleak outlooks described above. What is an example of enabling data models?

\section{A. The Sparse Synthesis Model}

The sparse synthesis signal model is one of the most celebrated data models. Many classes of signals, e.g., images, are observed to be very low-dimensional while their actual signal dimension can be quite high. This observation is modeled as follows: Any signal $\mathbf{x} \in \mathbb{R}^{d}$ of interest can be expressed as a linear combination of few columns of a fixed matrix $\mathbf{D} \in \mathbb{R}^{d \times n}$ called a dictionary. Typically, $n>d$ so that the model is rich enough to express interesting signals.

This model has been the focus of many papers, studying its core theoretical properties by exploring practical numerical algorithms for using it in practice (e.g. [1]-[3]), evaluating theoretically these algorithms' performance guarantees, addressing ways to obtain the dictionary from a bulk of data, and beyond all these, attacking a long series of applications in signal and image processing with this model, demonstrating often state-of-the-art results.

\section{B. The Cosparse Analysis Model}

The synthesis model has a 'twin' model that takes the analysis view point. Before introducing this analysis model, let us step back and look at a typical way the sparse synthesis model is used in a signal processing application. As described at the beginning, one may have an incomplete and/or noisecontaminated observation $\mathbf{y}$ of a signal $\mathbf{x}$. This means that there can be many - a lot of times, infinitely many-signals that could explain the observation $\mathbf{y}$ and hence it is difficult to recover $\mathbf{x}$. However, if we know that $\mathbf{x}$ is sparse in $\mathbf{D}$, then we can look for the coefficient vector $\mathbf{z}$ that is as sparse as possible while the resulting signal $\mathbf{D z}$ is 'compatible' with $\mathbf{y}$.

An interesting alternative has been used in practice successfully over the years; In this alternative, there is a fixed analysis operator $\Omega$ acting on the signal space, and one looks for the signal $\mathrm{x}$ that sparsifies the analysis representation $\Omega \mathbf{x}$ the most while being compatible with the observation $\mathbf{y}$, instead of searching for the sparsest synthesis representation $\mathbf{z}$ with $\mathbf{x}=$ Dz. Several works, e.g., [4]-[7], have empirically demonstrated the effectiveness of the analysis-based approaches and have shown either implicitly or explicitly the distinctness of two approaches (synthesis vs. analysis). On the other hand, perhaps due to the strong resemblance between the two methods, it was only recent that the underlying distinct model, which was named cosparse analysis model, was explicitly described and studied in [8].

In the cosparse analysis model, a signal $\mathbf{x} \in \mathbb{R}^{d}$ is said to be cosparse with respect to an analysis operator $\Omega \in \mathbb{R}^{p \times d}$ if the analysis representation $\Omega \mathbf{x}$ contains many zero entries. Further, the number of zeros $\ell=p-\|\boldsymbol{\Omega} \mathbf{x}\|_{0}$ is called the cosparsity of $\mathrm{x}$ and we say that $\mathrm{x}$ is $\ell$-cosparse. The index set where $\Omega \mathrm{x}$ is zero will be called the cosupport of $\mathrm{x}$. We want to bring to the reader's attention that we are focused on the fact that $\Omega \mathrm{x}$ has many zeros entries rather than few non-zero entries since there is generally a non-trivial lower limit on the number of non-zero entries [8]. 


\section{The Greedy Analysis Pursuit Algorithm}

Given an incomplete observation $\mathbf{y}$ of a cosparse signal $\mathbf{x}$, how to recover the original signal $\mathrm{x}$ ?

For this task, a greedy algorithm in the spirit of Matching Pursuit algorithms for the sparse synthesis model was proposed in [8] for the cosparse signal recovery and was named Greedy Analysis Pursuit (GAP). Further details are left to Section II and [8]. Let us now elaborate on the cosparse signal recovery problem for which the GAP algorithm was applied.

Let $\mathbf{x} \in \mathbb{R}^{d}$ be a cosparse signal with respect to an analysis operator $\Omega \in \mathbb{R}^{p \times d}$. Suppose that the only information available for $\mathbf{x}$, apart from that it is cosparse with respect to $\Omega$, is given by

$$
\mathbf{y}=\mathbf{M x},
$$

where $\mathbf{M} \in \mathbb{R}^{m \times d}$ is a measurement matrix. We assume that $m<d$, so the relation is underdetermined. Then, the cosparse signal recovery problem is the triplet $(\mathbf{y}, \mathbf{M}, \boldsymbol{\Omega})$ - possibly the quartet $(\mathbf{y}, \mathbf{M}, \boldsymbol{\Omega}, \ell)$, where $\ell$ is the coparsity of $\mathbf{x}$. The goal in the cosparse signal recovery is to recover $\mathbf{x}$.

The effectiveness of the GAP for the cosparse signal recovery was well-demonstrated in [8]. Surprsingly, the GAP was observed to outperform the analysis $\ell_{1}$-minimization algorithm.

\section{Contribution}

The type of cosparse recovery problem described above rarely occurs in practice. One issue is that the clean measurements/observations $\mathbf{y}=\mathbf{M x}$ of a cosparse signal $\mathbf{x}$ are unlikely to be available. For this reason, it is better to suppose that $\mathbf{y}=\mathbf{M x}+\mathbf{n}$ where $\mathbf{n}$ is a noise term.

Another problem of practical nature comes from the inexactness of the model. That is, most signals $\mathbf{x}$ of interest are actually not exactly cosparse. This leads us to work with the following approximate cosparse signal model: A signal $\mathbf{x}$ is approximately cosparse with respect to $\Omega$ if there is an exact cosparse signal $\mathrm{x}^{*}$ close to $\mathrm{x}$, i.e.,

$$
\mathrm{x} \approx \mathrm{x}^{*} \text { and } p-\left\|\boldsymbol{\Omega} \mathrm{x}^{*}\right\|_{0} \gg 1 .
$$

Due to these practical issues, though it has delivered encouraging empirical results, the GAP algorithm has not been a viable tool to be used directly in applications. The contribution of this paper is to adapt the GAP and make it able to perform stable cosparse signal reconstruction when the observation is contaminated by noise. This is empirically demonstrated on toy MRI problems.

\section{NOISELESS GAP ALGORITHM}

The Greedy Analysis Pursuit (GAP) algorithm as appeared in [8] is presented in this section.

To begin, we fix some notations. For an index set $I \subset \llbracket 1, p \rrbracket$, $\boldsymbol{\Omega}_{I}$ is the submatrix of $\boldsymbol{\Omega}$ that is obtained by retaining the rows of $\Omega$ indexed by $I$. A vector $\mathbf{x}_{0} \in \mathbb{R}^{d}$ is a cosparse signal with respect to $\Omega$ and will serve as the ground truth. The cosupport of $\mathbf{x}_{0}$ is denoted by $\Lambda$.
The GAP attempts to solve the linear inverse problem (1) where $\mathbf{y}:=\mathbf{M x}_{0}$. Its aim is to identify the cosupport $\Lambda$ and hence to pin down the signal $\mathbf{x}_{0}$. An interesting twist is that it tries to remove rows of $\Omega$ in a greedy way to arrive at $\Omega_{\Lambda}$ in the end. At the first step, the GAP takes $\hat{\Lambda}_{0}=\llbracket 1, p \rrbracket$ to be the initial estimate of the cosupport $\Lambda$ and removes elements from $\hat{\Lambda}_{0}$ to obtain a new estimate $\hat{\Lambda}_{1}$. What would be a good way to choose the elements to be discarded? If we pretend that we know $\mathbf{x}_{0}$, then this would be an easy task: We will compute $\boldsymbol{\Omega}_{\hat{\Lambda}_{0}} \mathbf{x}_{0}$ and choose any index that corresponds to a non-zero entry. Of course, we do not have $\mathrm{x}_{0}$ but only y. These arguments naturally suggest: First, obtain a 'good enough' estimate $\hat{\mathbf{x}}_{0}$ of $\mathbf{x}_{0}$ using $\mathbf{y}$. Then, compute $\boldsymbol{\Omega}_{\hat{\Lambda}_{0}} \hat{\mathbf{x}}_{0}$ and decide which elements to remove. For the first half of the task, we let

$$
\hat{\mathbf{x}}_{0}:=\underset{\mathbf{x}}{\operatorname{argmin}}\left\|\boldsymbol{\Omega}_{\hat{\Lambda}_{0}} \mathbf{x}\right\|_{2} \quad \text { subject to } \quad \mathbf{y}=\mathbf{M x} .
$$

For the second half, note first that most likely all elements of $\boldsymbol{\Omega}_{\hat{\Lambda}_{0}} \hat{\mathbf{x}}_{0}$ are non-zero. Hence, some care must be taken in choosing elements to remove. Assuming that $\hat{\mathbf{x}}_{0}$ is a good estimate of $\mathbf{x}_{0}$, a safe route would be to choose the entries of $\boldsymbol{\Omega}_{\hat{\Lambda}_{0}} \hat{\mathbf{x}}_{0}$ with the maximum magnitudes. We can see that this is a sensible approach once we view that $\boldsymbol{\Omega}_{\hat{\Lambda}_{0}} \hat{\mathbf{x}}_{0}$ is a 'small' perturbation of $\Omega_{\hat{\Lambda}_{0}} \mathbf{x}_{0}$. Thus, for the second part, we let

$$
\hat{\Lambda}_{1}:=\hat{\Lambda}_{0} \backslash\left\{\operatorname{argmax}\left\{\left|\omega^{j} \hat{\mathbf{x}}_{0}\right|: j \in \hat{\Lambda}_{0}\right\}\right\},
$$

where $\omega^{j}$ is the $j$-th row of $\boldsymbol{\Omega}$. The version of GAP alternates the two steps above until an appropriate stopping criterion is met.

\section{NeW Noise-Aware GAP Algorithm}

The new version of GAP attempts to solve the linear inverse problem with noise,

$$
\mathbf{y}=\mathbf{M x}+\mathbf{n}
$$

where $\mathbf{y}:=\mathbf{M x}_{0}+\mathbf{n}$ is the noisy measurements/observations of $\mathbf{x}_{0}$. We assume the knowledge of the noise level $\epsilon$; to be precise, $\|\mathbf{n}\|_{2} \leq \epsilon$.

Looking at how (1) is related to the estimate update step (2) of the original GAP, it is natural to consider the alternative

$$
\hat{\mathbf{x}}_{0}:=\underset{\mathbf{x}}{\operatorname{argmin}}\left\|\boldsymbol{\Omega}_{\hat{\Lambda}_{0}} \mathbf{x}\right\|_{2} \quad \text { subject to } \quad\|\mathbf{y}-\mathbf{M x}\|_{2} \leq \epsilon
$$

in order to adapt the GAP for the problem (4). The same cosupport estimate update step (3) can then be used. This leads to the new noise-aware version of GAP, called GAPn. The description of the algorithm is given in Algorithm 1.

a) Stopping Criteria: Various stopping criteria can be used to terminate the GAPn iterations. One criterion that proved to be useful was $\left\|\boldsymbol{\Omega}_{\hat{\Lambda}_{i}} \hat{\mathbf{x}}_{i}\right\|_{\infty}<\eta_{\text {coef }}$ where $\eta_{\text {coef }}$ is some prescribed (small) value. Another possibility is $\| \hat{\mathbf{x}}_{i}-$ $\hat{\mathbf{x}}_{i-1} \|_{2}<\eta_{\text {stable }}$ for some $\eta_{\text {stable }}>0$. One can also decide on a prescribed cosparsity as a stopping criterion: $\left|\hat{\Lambda}_{i}\right|<\ell_{\text {stop }}$. The first option was used in our experiment. 


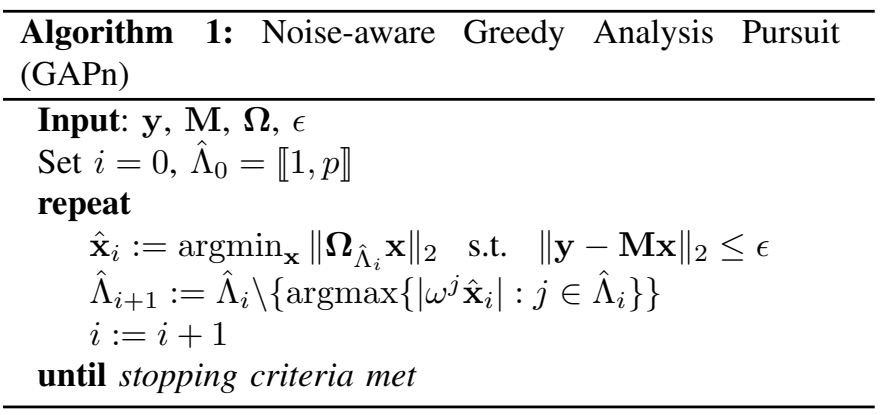

b) Solving the Inner Optimization (5): In the implementation of the GAPn, we solved the optimization (5) by solving an alternative problem (with conjugate gradient method):

$$
\hat{\mathbf{x}}_{0}^{\prime}:=\underset{\mathbf{x}}{\operatorname{argmin}}\|\mathbf{y}-\mathbf{M x}\|_{2}^{2}+\lambda\left\|\boldsymbol{\Omega}_{\hat{\Lambda}_{0}} \mathbf{x}\right\|_{2}^{2}
$$

for appropriate value $\lambda>0$. In fact, the value of $\lambda$ was varied until $\hat{\mathbf{x}}_{0}^{\prime}$ satisfies the constraint $\|\mathbf{y}-\mathbf{M x}\|_{2} \leq \epsilon$.

c) Super-greedy GAP Algorithms: Multiple rows of $\Omega$ can be removed at each cosupport update step. Two simple alternatives to (3) can be conceived. One way would be to discard the rows corresponding to $r$ largest analysis coefficients at each iteration for some fixed $r>1$. The second alternative is to perform

$$
\Gamma_{i}:=\left\{j \in \hat{\Lambda}_{i}:\left|\alpha_{j}\right| \geq t \max _{s \in \hat{\Lambda}_{i}}\left|\alpha_{s}\right|\right\}, \quad \hat{\Lambda}_{i+1}:=\hat{\Lambda}_{i} \backslash \Gamma_{i},
$$

where $\alpha:=\boldsymbol{\Omega}_{\hat{\Lambda}_{i}} \hat{\mathbf{x}}_{i}$, for some fixed $0<t<1$. The Noiseless GAP using this alternative was discussed in [8].

\section{EXPERIMENTS}

We show the experimental results obtained by applying the GAPn algorithm presented in Section III.

\section{A. Synthetic Cosparse Signal Recovery}

As a first demonstration, we conducted a synthetic experiment on cosparse signal recovery. In this experiment, we fixed the signal dimension $d=200$ and the size of the analysis operator $p=240$. Two values of measurement size were tried: $m=160$ and $m=80$. The cosparsity was also fixed at $\ell=180$ and $\ell=195$ respectively for the cases $m=160$ and $m=80$. Once $d, p, m$ and $\ell$ are fixed, the noise intensity $\epsilon_{\exp }$ were varied. At each noise intensity, the experiment was repeated 50 times and the average relative error was computed.

The details of how the cosparse signal recovery problem $(\mathbf{y}, \mathbf{M}, \boldsymbol{\Omega}, \epsilon)$ (noise intensity is added here.) was constructed are as follows: The entries of $\mathbf{M} \in \mathbb{R}^{m \times d}$ are simply drawn independently from the normal distribution. $\boldsymbol{\Omega}^{T} \in \mathbb{R}^{d \times p}$ was constructed to be a random tight frame with uniform column norm. Random $\ell$ elements of $\llbracket 1, p \rrbracket$ were selected to be a set $\Lambda$. A Gaussian random signal in the null space of $\boldsymbol{\Omega}_{\Lambda}$ was constructed to be $\mathbf{x}_{0}$. A noise term $\mathbf{n}$ with $\|\mathbf{n}\|_{2}=\epsilon_{\exp }$ was constructed and finally the observation $\mathbf{y}$ was computed by $\mathbf{y}=\mathbf{M x}_{0}+\mathbf{n}$. The noise level $\epsilon:=1.01 \epsilon_{\exp }$ was passed to the algorithms.

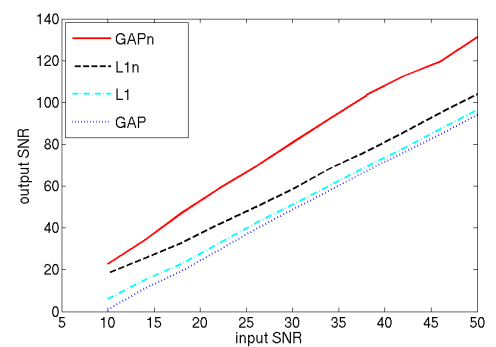

Fig. 1. Output SNR $20 \log _{10}\left(\left\|\mathbf{x}_{0}\right\|_{2} /\left\|\hat{\mathbf{x}}-\mathbf{x}_{0}\right\|_{2}\right)$ as a function of input SNR $20 \log _{10}\left(\left\|\mathbf{M} \mathbf{x}_{0}\right\|_{2} / \epsilon_{\exp }\right)$ for the tested algorithms for the case $d=200$, $p=240, m=160$, and $\ell=180$.

The results for the first case is shown in Figure 1. In addition to the GAPn, the analysis $\ell_{1}$ which solves

$$
\hat{\mathbf{x}}:=\underset{\mathbf{x}}{\operatorname{argmin}}\|\boldsymbol{\Omega} \mathbf{x}\|_{1} \quad \text { subject to } \quad\|\mathbf{y}-\mathbf{M x}\|_{2} \leq \epsilon,
$$

was tested. In the figure, the label L1 corresponds to the result when $\epsilon$ was taken to be 0 above and the label L1n corresponds to the one where $\epsilon$ was set at the value described above. GAP denotes the noiseless version. The figure shows that while the GAP performs worse than the analysis $\ell_{1}$, the noise-aware GAPn outperforms the noise-aware $\ell_{1}$ in the considered SNR range. The GAPn was also observed to work better than the $\ell_{1}$ in the setting $d=200, p=240, m=160$, and $\ell=195$, but the result is not shown here in the interest of space.

\section{B. Shepp-Logan Phantom Reconstruction}

As a more realistic test, we applied the GAPn for the Shepp Logan phantom reconstruction problem. In this experiment, the $256 \times 256$ phantom image was chosen and the measurements were made along 22 radial lines in the 2D Fourier domain. The observation was further corrupted by Gaussian noise; The noise level was $\epsilon_{\exp }=0.05\left\|\mathbf{M x}_{0}\right\|_{2}$ and $\epsilon=1.01 \epsilon_{\exp }$, using the notations of the previous subsection.

We have tested the following algorithms: tvdantzig logbarrier and tvqc_logbarrier from 11 magic-1.1 package, and the GAPn. For the GAPn, the analysis operator used was the one-step finite difference operator $\boldsymbol{\Omega}_{\mathrm{DIF}}$ that consists of all the horizontal and vertical differences of neighboring pixels.

Figure 2 demonstrates that the GAPn result captures the piece-wise constant nature of the original image better than tvdantzig or tvqc results.

\section{MRI Image Reconstruction}

The next experiment is related to the issue of approximate signal model. Namely, the signals we meet in practice are not exactly cosparse. For this experiment, we have chosen a (crop of) MRI image generated from the FSL MNI152 T1 $0.5 \mathrm{~mm}$ image data. ${ }^{1}$ The setting of the problem is similar to the previous subsection, and the measurements of the image are obtained along 50 radial lines in the Fourier domain. However, no intentional noise was added.

\footnotetext{
${ }^{1}$ Available from Debian Linux package fsl-mni152-templates.
} 

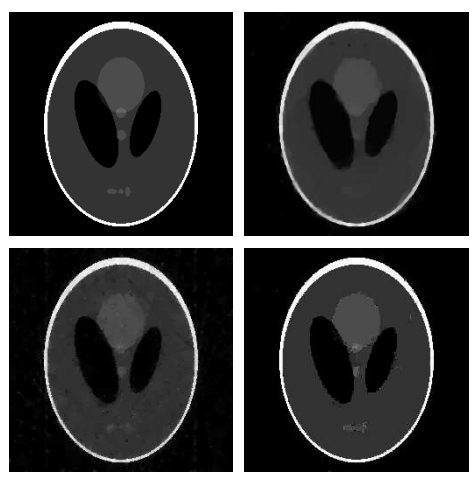

Fig. 2. Shepp Logan phantom reconstruction results from 22 radial observation lines with $5 \%$ noise corruption. The original phantom (top left), tvdantzig (top right), tvqc (bottom left), and the GAP (bottom right).
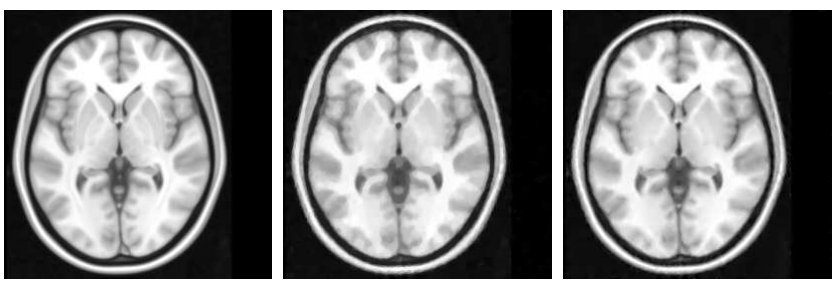

Fig. 3. MRI reconstruction results from 50 radial observation lines. The original (left), TV-min. (center), and GAPn (right) reconstructed images.

\section{1) Cosparsity with One-step Finite Difference Operator:}

The image is not seen to be truely cosparse with respect to $\boldsymbol{\Omega}_{\mathrm{DIF}}$. Given this, we can envision two possible ways to reconstruct the target image. One approach is to treat the inexactness of the signal to the model as noise. Here, we start with an implicit assumption that there is a signal $\mathbf{x}^{*}$ which is cosparse with respect to $\Omega_{\mathrm{DIF}}$ and is nearby the target signal $\mathbf{x}$. Then, we recognize the problem as solving (4) with noise term given by $\mathbf{n}=\mathbf{M}\left(\mathbf{x}-\mathbf{x}^{*}\right)$, and try to recover $\mathbf{x}^{*}$. This requires the 'noise' intensity to be estimated.

The other strategy is to keep the constraint (1) since the observation $\mathbf{y}$ is thought to be clean and to apply the GAP with noise level 0 (or very small value). The algorithm stops when $\left\|\boldsymbol{\Omega}_{\hat{\Lambda}_{i}} \hat{\mathbf{x}}_{i}\right\|_{\infty}$ is deemed to be relatively small.

The resconstruction results showed that the total-variation minimization algorithms of 11 magic -1.1 give better output than the GAP algorithm with analysis operator $\Omega_{\mathrm{DIF}}$ (result not displayed). Visually, all those results suffer from staircasing artifacts related to either 'TV-norm' or the one-step finite difference operator. The reconstruction from the GAP showed a bit more pronounced blockiness, which indicates that the GAP is enforcing the cosparse analysis model more strongly. The differences between the two approaches described above for handling the model mismatch were not significant.

2) Cosparsity with Second-order Differences: Interestingly, when the one-step difference operator was replaced by the operator $\Omega_{\mathrm{D} 2}$ consisting of second-order differences in the horizontal, vertical, diagonal, and anti-diagonal directions, the reconstruction result from the GAPn with $\epsilon=10^{-3}\|\mathbf{y}\|_{2}$ and $\eta_{\text {coef }}=5 \cdot 10^{-4}$ was the best. Note that the signal $\mathbf{x}$ was normalized to take values in the interval $[0,1]$. The improvement on SNR was from 21.0dB $\left(\boldsymbol{\Omega}_{\mathrm{DIF}}\right)$ to $29.6 \mathrm{~dB}$ $\left(\Omega_{\mathrm{D} 2}\right)$ which was also better than $28.4 \mathrm{~dB}$ of the TV-based reconstruction. Perhaps more importantly, visual artifacts were significantly less pronounced. This shows the importance of using an appropriate analysis operator to fit the signals to the cosparse model. The original, and the reconstructed images using the TV-minimization and the GAPn with $\Omega_{\mathrm{D} 2}$ are shown in Figure 3.

\section{CONCLUSION}

In this work, we have extended the Greedy Analysis Pursuit algorithm of [8] so that it can be used for the reconstruction of cosparse signals when the noise term is present or for the reconstruction of signals that are approximately cosparse, in the context of linear inverse problems. The effectiveness of the proposed algorithm was demonstrated by experiments. The experimental results show that the GAPn algorithm outperforms the analysis $\ell_{1}$-minimization given the same knowledge on the noise intensity when the cosparse analysis model fits the signals of interest well. However, they also exposed the natural weakness of the algorithm when the model is not a good fit.

The weakness calls for the need to better design or learn analysis operators for signals of certain class. This need was confirmed by some improvements observed in our experimental results. The encouraging performance in this work prompts us to launch further studies: theoretical investigation of the behaviors related to the cosparse model and the algorithms; fine tuning the GAPn for the approximate cosparse model; and extensive comparisons with state-of-the-art sparse recovery algorithms (e.g., TVAL3 or ISD [9]).

\section{ACKNOWLEDGMENT}

This work was supported in part by the EU through the project SMALL (Sparse Models, Algorithms and Learning for Large-Scale data), FET-Open programme, under grant number: 225913.

\section{REFERENCES}

[1] S. Chen, D. Donoho, and M. Saunders, "Atomic decomposition by basis pursuit," SIAM J. Sci. Comput., vol. 20, pp. $33-61,1998$.

[2] S. Mallat and Z. Zhang, "Matching pursuits with time-frequency dictionaries," Signal Processing, IEEE Transactions on, vol. 41, p. 3397 3415, 1993.

[3] E. Candès and T. Tao, "The Dantzig selector: statistical estimation when $\mathrm{p}$ is much larger than n," Annals of Statistics, vol. 35, no. 6, pp. 2313-2351, 2007.

[4] M. Elad, P. Milanfar, and R. Rubinstein, "Analysis versus synthesis in signal priors," Inverse Problems, vol. 23, no. 3, pp. 947-968, June 2007.

[5] J. Portilla, "Image restoration through 10 analysis-based sparse optimization in tight frames," Proceedings of the 16th IEEE International Conference on Image Processing, pp. 3865-3868, 2009.

[6] I. W. Selesnick and M. A. T. Figueiredo, "Signal restoration with overcomplete wavelet transforms: Comparison of analysis and synthesis priors," In Proceedings of SPIE, vol. 7446 (Wavelets XIII), August 2009.

[7] J.-F. Cai, S. Osher, and Z. Shen, "Split bregman methods and frame based image restoration," Multiscale Modeling \& Simulation, vol. 8, no. 2, pp. 337-369, 2009

[8] S. Nam, M. E. Davies, M. Elad, and R. Gribonval, "The Cosparse Analysis Model and Algorithms," Research Report, 2011, submitted. [Online]. Available: http://hal.inria.fr/inria-00602205/en/

[9] Y. Wang and W. Yin, "Sparse signal reconstruction via iterative support detection," SIAM J. Imaging Sciences, vol. 3, no. 3, pp. 462-491, 2010. 